\title{
Structure determination of small molecule compounds by an electron diffractometer Synergy-ED
}

\author{
Sho Ito, ${ }^{a}$ Fraser J. White, ${ }^{b}$ Eiji Okunishi, ${ }^{c}$ Yoshitaka Aoyama, ${ }^{c}$ Akihito Yamano, ${ }^{a}$ \\ Hiroyasu Sato, ${ }^{a}$ Joseph D. Ferrara, ${ }^{d}$ Michał Jasnowski ${ }^{e}$ and Mathias Meyer ${ }^{\mathrm{e}}$ \\ aRigaku Corporation, 3-9-12 Matsubara-cho, Akishima, Tokyo 196-8666, Japan \\ ${ }^{b}$ Rigaku Europe SE, Hugenottenallee 167, 63263 Neu Isenburg, Germany \\ cJEOL Ltd, 3-1-2 Musashino, Akishima, Tokyo196-8558, Japan \\ dRigaku Americas Corporation, Inc., 9009 New Trails Drive, TheWoodlands, TX 77381-5209, USA \\ eRigaku Polska Sp. Z o.o., ul. Szarskiego 3, 54-609 Wrocław, Poland
}

\section{Abstract}

Micro Electron Diffraction (MicroED) has extended the limits of crystallography by enabling the determination of three dimensional molecular structures from sub- $\mu \mathrm{m}$ microcrystals. However, microED measurements using current state-of-the-art electron microscopes require experts in both electron microscopy and crystallography making the method rather difficult for researchers who simply need structures. Here, we present a diffractometer specifically designed for microED and show how it works for determining crystal structures. The newly developed electron diffractometer will provide many researchers with an easy path to structure determination of crystals that are less than $1 \mu \mathrm{m}$ in size

\section{Introduction}

X-ray crystallography has long been used as a powerful technique to determine the three dimensional molecular structure of small molecule compounds and biological macromolecules at atomic resolution. We have developed a series of X-ray diffractometers named XtaLAB, which enables researchers to provide structures of crystals of a few microns in size. Although X-ray crystallography is a very powerful technique, it requires crystals that are at least $1 \mu \mathrm{m}$ in size. Crystallization involves a lot of trial and error to determine the best conditions. Furthermore, crystallization is not always successful. Much effort has been expended to accelerate the determination of crystal structures, including the construction of synchrotron radiation facilities and the development of the crystalline sponge method (Hoshino et al., 2016; Inokuma et al., 2013). However, there are expected to be numerous crystal structures of many important compounds still remaining unknown due to this obstacle. In recent years, electron diffraction of microcrystals, called microED, has rapidly come into use (Shi et al., 2016; Sawaya et al., 2016; Jones et al., 2018; Nannenga, 2020) because the interaction between electrons and materials is much stronger than that of X-rays, therefore, the difficulty of crystallization can be mitigated; microED can determine the molecular structure of crystals smaller 
than $1 \mu \mathrm{m}$, which are difficult to measure routinely even with synchrotron undulator beamlines. For instance, the structure of lomaiviticin (Kim et al., 2021), which could not be determined by X-ray diffraction for twenty years, was determined by microED.

Although microED is becoming more widely known and used, the technique still requires two experts, one in electron microscopy and the other in crystallography. Furthermore, to the best of our knowledge, almost all microED datasets are collected using cryo-EM optimized to perform single particle analysis of macromolecules, and not dedicated to microED experiments. Therefore, the current problem is that it is not easy to perform microED experiments. We have developed an instrument specifically for microED measurements, "XtaLAB Synergy-ED", to streamline microED measurements. The XtaLAB Synergy-ED has been thoroughly tested in the course of development and has successfully produced crystal structures of a variety of compounds. In this paper, we report the configuration of the Synergy-ED coupled with CrysAlis ${ }^{\text {Pro }}$ for ED software, giving a seamless workflow from data collection, structure determination, and the results of structural analysis.

\section{Methods}

\subsection{Configuration of Synergy-ED}

The configuration of the newly developed Synergy-ED is briefly described below. The most important components are the lens system, the HyPix-ED, a direct electron detector, and CrysAlis ${ }^{\text {Pro }}$ for ED, the software controlling these components. 1. Lens system; a common lens system used in electron microscopes was adopted for Synergy-ED (Figure 1b). This system uses a fixed lens setting for electron diffraction in the electron microscope. When searching the target area, the image of the specimen is obtained on the HyPix-ED by changing the current of the intermediate lens 1. 2. HyPix-ED; direct electron detection of diffraction spots by HPC detectors such as HyPix-ED allows for very precise measurements. The detector is also very robust and can make measurements even when the primary beam is directly incident on the detector. In fact, all the measurements in this study were performed without the use of a direct beam stop. In addition, due to the high dynamic range of the detector (up to 31 bits), saturated diffraction spots rarely occur even in measurements that require long exposure time due to poor crystallinity of the crystals. 3. CrysAlis ${ }^{\text {Pro }}$ for ED; the most important component of Synergy-ED is CrysAlis ${ }^{\text {Pro }}$ for ED, the integrated control and processing software. This software performs stage control, diffraction experiments, data integration, and reduction. In the microED experiment, it is necessary to switch between image and diffraction modes to confirm the position and crystallinity of the crystals. With Synergy-ED, this switching can be performed with a single mouse click, which greatly reduces the burden on researchers. The data merging of crystals belonging to the space

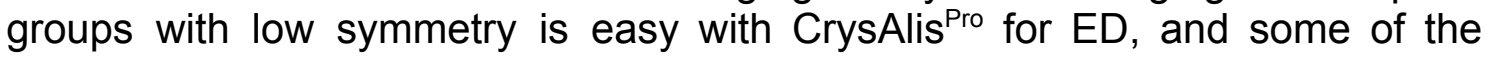
samples in this study were merged using this function. 


\subsection{Sample preparation and diffraction experiment}

All compounds used in this study were purchased from Tokyo Chemical Industry Co., Ltd, FUJIFILM Wako Pure Chemical Corporation, or Nacalai Tesque. Table 1 summarizes compounds subjected to the structure determinations in this study.

For all compounds except gefitinib, iron(II) phthalocyanine bis(pyridine) complex, diosgenin, and florasulam, purchased samples were used as received. Gefitinib and iron(II) phthalocyanine bis(pyridine) complex were measured from re-crystallized samples. Diosgenin and florasulam were crystallized directly on the grid (Figure. 2b). Specifically, $1 \mathrm{mg}$ of the compound was dissolved in $500 \mu \mathrm{L}$ of ethanol. Then, $1.5 \mu \mathrm{L}$ of the solutions were added to each grid and left for 10 min to dry, producing microcrystals of the compounds. Equal amounts of $\mathrm{TiO}_{2}$ rutile and anatase were mixed and deposited on a grid to determine if crystal polymorphs could be detected. The measurement process can be briefly described as follows (See also Figure 2a): 1. Spread the microcrystals of the compound on a grid. A pair of microscope glass slides was used to crush the samples to reduce the grain size when the average crystal size was larger than $1 \mu \mathrm{m}$. Importantly, the thickness of the crystal is more important than its size. Even if the crystal size itself is large, it can be measured if the thickness (the distance through which the electron beam penetrates the crystal) is less than $1 \mu \mathrm{m}$. 2. Install the grid into the electron diffractometer. 3. Locate the crystals in imaging mode and find the eucentric position (a step known to X-ray crystallographers as centering). 4. Irradiate the crystal with an electron beam and measure the diffraction intensity by the rotation method.

(a)

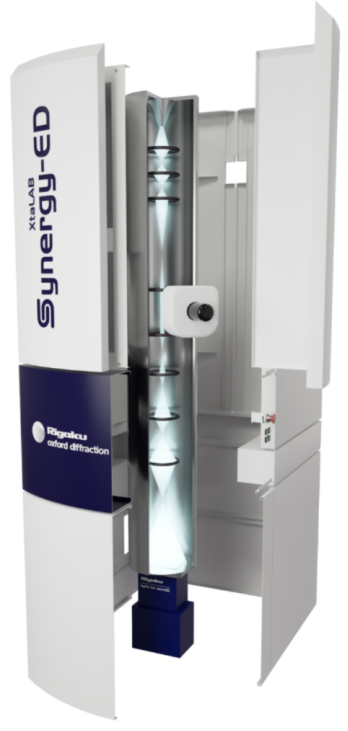

(b)

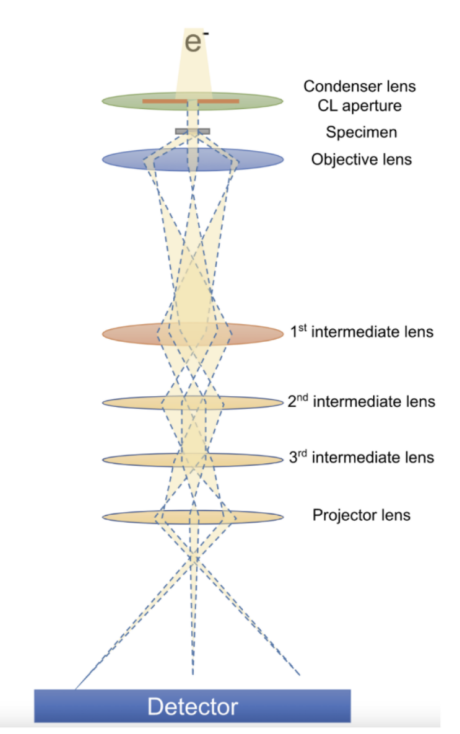

(c)

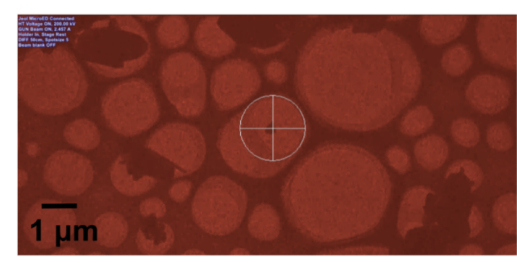

Change image/diffraction mode by clicking a button

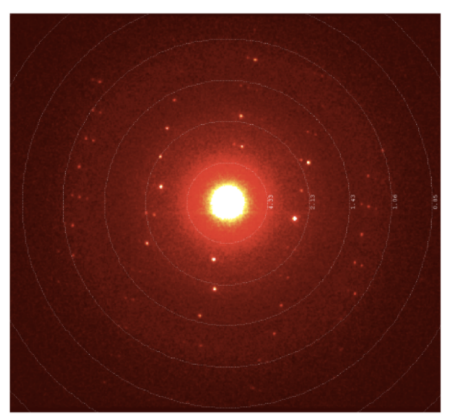


Figure. 1 Electron diffractometer specifically designed for microED experiments (a) Electron diffractometer used for the measurements (b) Lens system in diffraction mode (c) CrysAlis ${ }^{\text {Pro }}$ for ED allows easy switching between the image mode and the diffraction mode. Finding the eucentric is performed in image mode, and then the crystal is irradiated with an electron beam in diffraction mode to confirm the crystallinity of the crystal. If diffraction is observed, data collection commences. Diffraction mode of CrysAlis ${ }^{\text {Pro }}$ for ED: The diffraction pattern of L-tyrosine is shown as an example. The diffraction spots were observed to $0.84 \AA$ resolution (See also Table 1). Since the beam stop was not used in this study, a very intense white spot was observed in the center of the figure (primary beam).

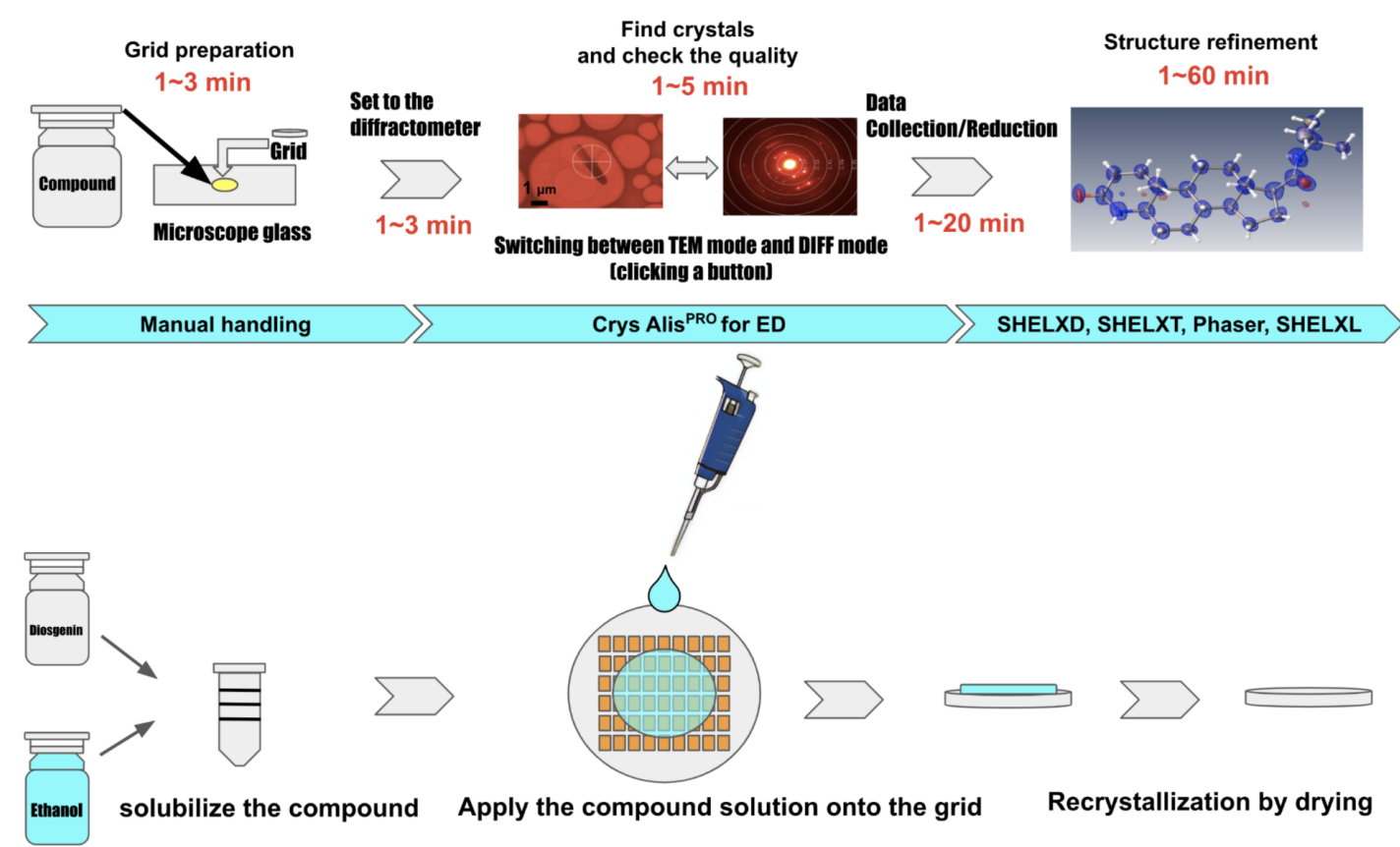

Figure. 2 Flow of structural analysis by microED

(a) Flow from sample preparation to structural analysis. For samples with high crystallinity, the crystals are loaded directly into the grid. The amount of sample required is less than $1 \mathrm{mg}$, and the crystals are scattered on the grid by simply placing the grid on the crystals placed on a microscope glass. Instrument control, data acquisition, and data reduction are all performed by CrysAlis ${ }^{\text {Pro }}$ for ED. Phasing and refinement are performed in the conventional way used in X-ray crystallography. (b) Improvement of crystallinity by on-grid re-crystallization for samples with poor crystallinity, re-crystallization on-grid may improve the crystallinity. Dissolve the compound in some solution, load a few $\mu \mathrm{L}$ of the solution onto the grid, and dry the solution.

\subsection{Diffraction experiment and structure analysis}

A total of 14 compounds were measured using the electron diffractometer. The measured samples are listed in Table 1. For the electron beam, the accelerating voltage was set to $200 \mathrm{kV}$ (corresponding to a wavelength of $0.0251 \AA$ ). The 
total rotation range depends on the compound and the position on the grid (the maximum rotation range is $-80^{\circ}$ to $+80^{\circ}$ ), but it was typically $-45^{\circ}$ to $+45^{\circ}, 90^{\circ}$ in total. The exposure time was set between 1 and $10 \mathrm{~s}$ so that the corresponding frame rate ranged between $0.1 \mathrm{~Hz}$ and $1.0 \mathrm{~Hz}$. To reduce the radiation damage to the crystals, the dose rate was set to ca. $0.01 \mathrm{e}^{-} / \AA^{2} / \mathrm{s}$. For the rutile and anatase of $\mathrm{TiO}_{2}$, only the determination of the unit cell (i.e., indexing) was performed. Intensity integration, scaling, and merging were performed by CrysAlis $^{\text {Pro }}$ for ED. In the case of crystals with low symmetry, such as triclinic, multiple datasets were merged so that the data completeness exceeded $70 \%$. Apart from diosgenin, the initial phases for each compound were determined by SHELXD or SHELXT (Schneider \& Sheldrick, 2002; George M. Sheldrick, 2015). For the determination of the initial phases of diosgenin, the molecular replacement program in Phaser was used with CCDC 1522829 as a search model. The structure refinement was performed by the full matrix least-squares method of SHELXL (George M. Sheldrick, 2015). The SFAC instructions were used to input atomic scattering factors for electrons (Peng, 1999) to replace those for X-rays.

\section{Results and Discussion}

\subsection{Crystal structures determined with Synergy-ED}

The results of structural analysis are shown in Table 1 and Figure 3 . All datasets were collected at least to $1.2 \AA$ resolution. The resolution of $1.2 \AA$ is derived from Sheldrick's rule (G. M. Sheldrick, 1990) and is the minimum resolution for which direct methods/intrinsic phasing are applicable. The statistics of all datasets were appropriate for microED data measurements. Multiple datasets were merged for gefitinib, iron(II) phthalocyanine bis(pyridine) complex, 2,4,6-Tri(4-pyridyl)-1,3,5-triazine, 1,3,5-Triphenylbenzene, acetaminophen, florasulam, and diosgenin to improve data completeness. The $R_{\text {int }}$ and $R_{\text {pim }}$ values did not deteriorate severely after data merging, suggesting not only high isomorphism of the crystals but also the high accuracy of the measurements from the diffractometer. The crystal with the lowest symmetry among the crystals measured in this study was the $P \overline{1}$ of gefitinib. Five datasets were merged to increase the completeness of the data. After merging, the data completeness reached $86 \%$, which can be considered to be high for a crystal of

$P \overline{1}$. On the other hand, for crystals such as D-glucose and L-tyrosine, which crystallize in the space group of $P 2_{1} 2_{1} 2_{1}$ and $\mathrm{K}_{2} \mathrm{PtCl}_{4}$, tetragonal crystal, it was possible to collect datasets with completeness above $80 \%$ from one crystal. The results show that crystals belonging to a crystal system with higher symmetry than the orthogonal system can obtain sufficient completeness from one crystal. In addition, the anisotropic displacement parameter remained positive definite for all structures, which indicates that the data collection and refinement were performed successfully. The shortest time taken to load a sample on the grid and finish data collection was about 5 minutes, and even the most time-consuming compound took about 30 minutes. The sample holder used in 
this measurement can take only one grid at a time, therefore, the measurement can be further accelerated by using a sample holder that can store multiple grids at once. Importantly, two different unit cells were observed in the $\mathrm{TiO}_{2}$ sample (Figure. 4), indicating that rapid measurement using microED is also effective in detecting polymorphism and quantitative analysis.

Table 1 Data collection and refinement statistics

\begin{tabular}{|c|c|c|c|c|c|c|c|}
\hline Compound & Gefitinib & D-glucose & L-tyrosine & Iron(II) phthalocyanine bis(pyridine) complex & 2,4,6-Tri(4-pyridyl)-1,3,5-triazine & Cytidine & Acetaminophen \\
\hline Merged crystals & 5 & 1 & 1 & 4 & 3 & 1 & 7 \\
\hline Resolution & $12.87-0.98$ (1.01-010.98) & $15.27-0.90(0.944-0.90)$ & $21.59-0.837(0.87-0.84)$ & $19.99-0.90(0.93-0.90)$ & $10.46-0.90(0.93-0.90)$ & $14.60-0.95(0.98-0.95)$ & $11.99-0.900(0.93-0.90)$ \\
\hline Reflections & $7301(756)$ & $1536(170)$ & $2546(270)$ & $14611(1541)$ & $3614(286)$ & $2411(270)$ & $5392(528)$ \\
\hline Unique Reflections & $2129(208)$ & $557(65)$ & $855(87)$ & $2435(251)$ & $996(95)$ & $699(72)$ & $1303(129)$ \\
\hline Unit Cell & $\begin{array}{l}9.146(4), 9.990(5), 13.028(5) \\
93.78(4), 97.00(4), 101.72(4)\end{array}$ & $5.177(2), 10.691(5), 15.272(7)$ & $5.96(9), 7.09(14), 21.59(15)$ & $\begin{array}{l}9.25(12), 19.99(19), 9.66(10) \\
111.6(6)\end{array}$ & $\begin{array}{r}13.507(10), 11.432(7), \\
11.114(9)\end{array}$ & $5.1(5), 13.8(3), 14.6(3)$ & $\begin{array}{l}7.344(5), 9.645(6), 12.087(7) \\
97.37(5)\end{array}$ \\
\hline Space group & $P-1$ & $P 2,2_{2} 2_{1}$ & $P 22_{1} 2_{1}$ & $P 2,1 c$ & $c 2 / c$ & $P 2,2,2_{1}$ & $P 2, l_{n}$ \\
\hline Completeness & $83.0(79.7)$ & $73.5(78.3)$ & $85.2(84.5)$ & $98.0(99.2)$ & $81.0(77.2)$ & $87.7(83.7)$ & $99.4(97.7)$ \\
\hline Usig(I) & $19.30(1.81)$ & $13.12(2.13)$ & $27.57(7.74)$ & $8.37(3.61)$ & $29.16(5.45)$ & $5.11(2.12)$ & $22.38(4.09)$ \\
\hline Redundancy & $3.4(3.6)$ & $2.8(2.6)$ & $3.0(3.1)$ & $6.0(6.2)$ & $3.6(3.0)$ & $3.4(3.8)$ & $4.1(4.1)$ \\
\hline Rint & $9.5(59.0)$ & $11.1(41.4)$ & $10.7(31.8)$ & $18.1(42.5)$ & $8.9(32.2)$ & $15.4(48.9)$ & $8.8(37.8)$ \\
\hline Rpim & $6.3(35.2)$ & $8.4(33.5)$ & $7.9(21.2)$ & $7.6(17.3)$ & $5.4(23.6)$ & $9.9(28.9)$ & $5.9(21.0)$ \\
\hline $\mathrm{cc}_{1 / 2}$ & $0.996(0.660)$ & $0.991(0.368)$ & $0.985(0.730)$ & $0.991(0.881)$ & $0.996(0.878)$ & $0.983(0.655)$ & $0.992(0.859)$ \\
\hline R1 & 15.45 & 11.94 & 13.63 & 16.59 & 19 & 9.88 & 12.02 \\
\hline wR2 & 36.97 & 34.69 & 32.35 & 36.04 & 43.28 & 26.34 & 33.94 \\
\hline Goof & 2.167 & 1.156 & 1.116 & 1.276 & 2.477 & 1.044 & 1.074 \\
\hline Compound & Finasteride & $\mathrm{K}_{2} \mathrm{PtCl}_{4}$ & Florasulam & ZIF-8 & Rubrene & 1,3,5-Triphenylbenzene & Diosgenine (not deposited) \\
\hline Merged crystals & 1 & 1 & 10 & 1 & 1 & 1 & 3 \\
\hline Resolution & $\begin{array}{l}26.77-0.95(0.99-0.95) \\
5725\end{array}$ & $7.07-0.84(0.89-0.85)$ & $9.59-0.95(0.98-0.95)$ & 12.37-1.00 (1.05-1.00) & $14.16-0.90(14.16-0.90)$ & 20.24-1.00 (1.04-1.00) & $\begin{array}{l}34.06-1.10(1.14-1.10) \\
20271(246)\end{array}$ \\
\hline $\begin{array}{l}\text { Reflections } \\
\text { Unique Reflections }\end{array}$ & & $\begin{array}{l}423(83) \\
113(17)\end{array}$ & $12142(1341)$ & $\begin{array}{l}2256(290) \\
308(38)\end{array}$ & $\begin{array}{l}4535(497) \\
954(101)\end{array}$ & $\begin{array}{l}3427(374) \\
932 \text { (96) }\end{array}$ & $\begin{array}{l}20271(246) \\
2004(211)\end{array}$ \\
\hline Unique Reflections & $\begin{array}{c}1418(146) \\
6.66(19), 13.2(3), 26.77(12)\end{array}$ & $\begin{array}{c}113(17) \\
7.07(11), 4.16(16)\end{array}$ & $\begin{array}{c}1506(152) \\
8.302(3), 10.025(6), 10.638(6)\end{array}$ & $\begin{array}{l}308(38) \\
17.497(5)\end{array}$ & $\begin{array}{c}954(101) \\
26.34(10), 7.03(10), 14.16(16)\end{array}$ & $\begin{array}{c}932(96) \\
7.8(5), 20.2(2), 11.5(2)\end{array}$ & $\begin{array}{c}2004(211) \\
7.6,20.35,34.06\end{array}$ \\
\hline Unit Cell & $\begin{array}{c}6.66(19), 13.2(3), 26.77(12) \\
P 21_{1}, 2_{1}\end{array}$ & $\begin{array}{c}7.07(11), 4.16(16) \\
P 4 / m m m\end{array}$ & 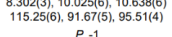 & $17.497(5)$ & $26.34(10), 7.03(10), 14.16(16)$ & $7.8(5), 20.2(2), 11.5(2)$ & $\begin{array}{c}7.6,20.35,34.06 \\
P 2,2,2,\end{array}$ \\
\hline $\begin{array}{l}\text { Space group } \\
\text { Completeness }\end{array}$ & $\begin{array}{c}P 2,2,2,1 \\
82.8(84.4)\end{array}$ & $\begin{array}{l}P 4 / m m m \\
84.3(100.0)\end{array}$ & $\begin{array}{c}P-1 \\
77.5(77.6)\end{array}$ & $\begin{array}{c}1-43 m \\
97.2(100.0)\end{array}$ & $\begin{array}{c}\text { C mce } \\
86.0(84.9)\end{array}$ & $\begin{array}{c}P n a 2_{1} \\
80.9(82.1)\end{array}$ & $\begin{array}{c}P 2,2,2,2 \\
81.8(85.8)\end{array}$ \\
\hline $\begin{array}{c}\text { Completeteness } \\
\text { Isig(I) }\end{array}$ & $14.42(2.42)$ & $43.95(20.42)$ & $14.48(3.43)$ & $\begin{array}{l}97.2(100.0) \\
11.39(1.41)\end{array}$ & $5.96(2.57)$ & $6.86(1.50)$ & 14.26 (3.7.79) \\
\hline Redundancy & $3.8(4.1)$ & $3.7(4.9)$ & $8.1(8.8)$ & $7.3(7.6)$ & $4.8(4.9)$ & $3.7(3.9)$ & $10.1(11.0)$ \\
\hline Rint & $14.9(59.0)$ & $6.5(12.0)$ & $19.5(64.1)$ & $20.8(78.1)$ & $18.6(46.3)$ & $19.1(59.0)$ & $20.9(65.5)$ \\
\hline Rpim & $9.0(31.5)$ & $4.5(6.1)$ & $7.2(21.8)$ & $8.7(30.1)$ & $9.4(23.5)$ & $11.7(34.1)$ & $7.3(19.7)$ \\
\hline $\mathrm{cc}_{1 / 2}$ & $0.989(0.450)$ & $0.976(0.970)$ & $0.992(0.570)$ & $0.993(0.611)$ & $0.983(0.793)$ & $0.986(0.546)$ & $0.989(0.655)$ \\
\hline R1 & 13.51 & 16.52 & 16.02 & 11.37 & 9.2 & 10.67 & 21.2 \\
\hline wR2 & 30.83 & 44.66 & 37.69 & 31.36 & 24.52 & 30.98 & 52.43 \\
\hline Goof & 1.086 & 3.167 & 2.269 & 1.089 & 1.111 & 1.031 & 1.862 \\
\hline
\end{tabular}
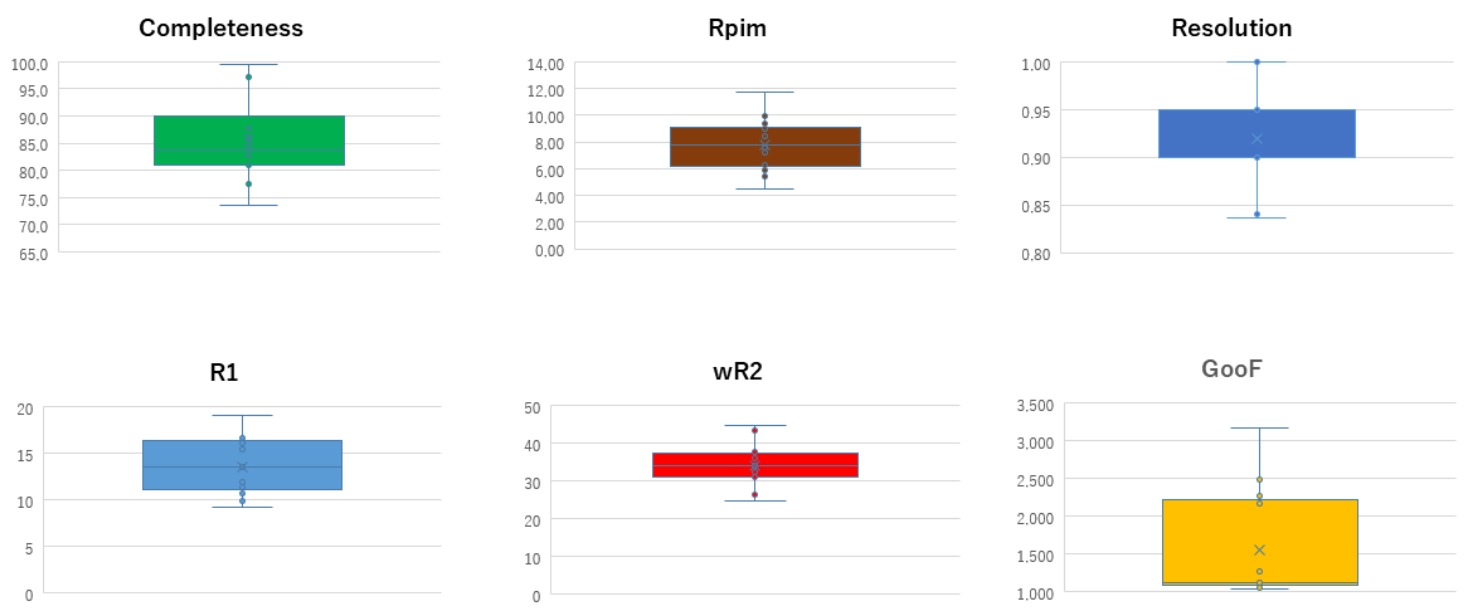

Figure 3 Distribution of statistical values of measured data 
(a)
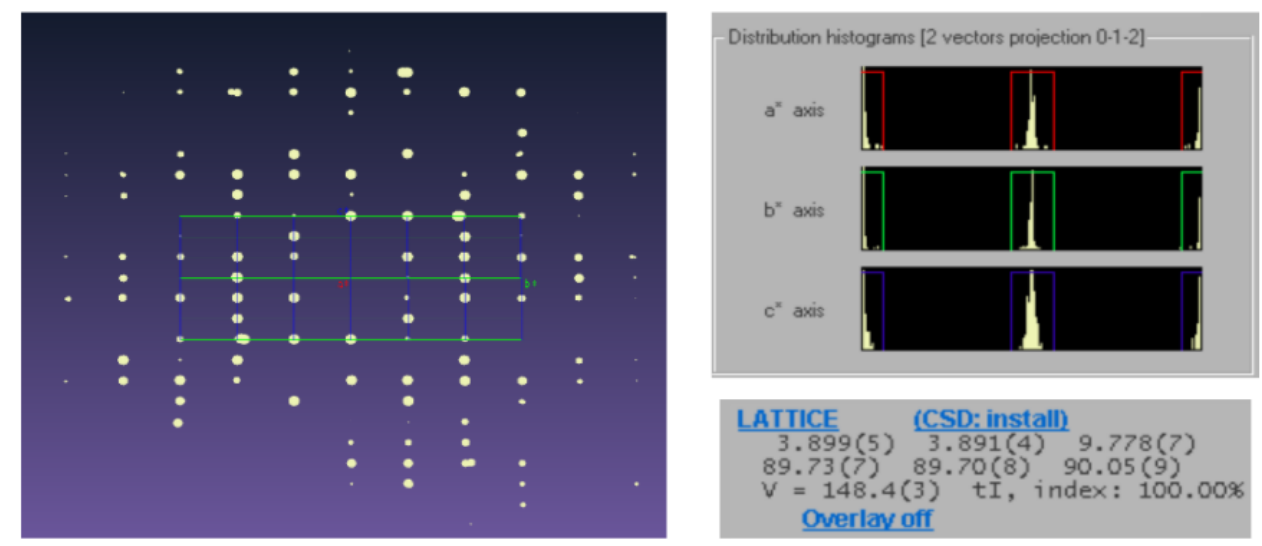

(b)
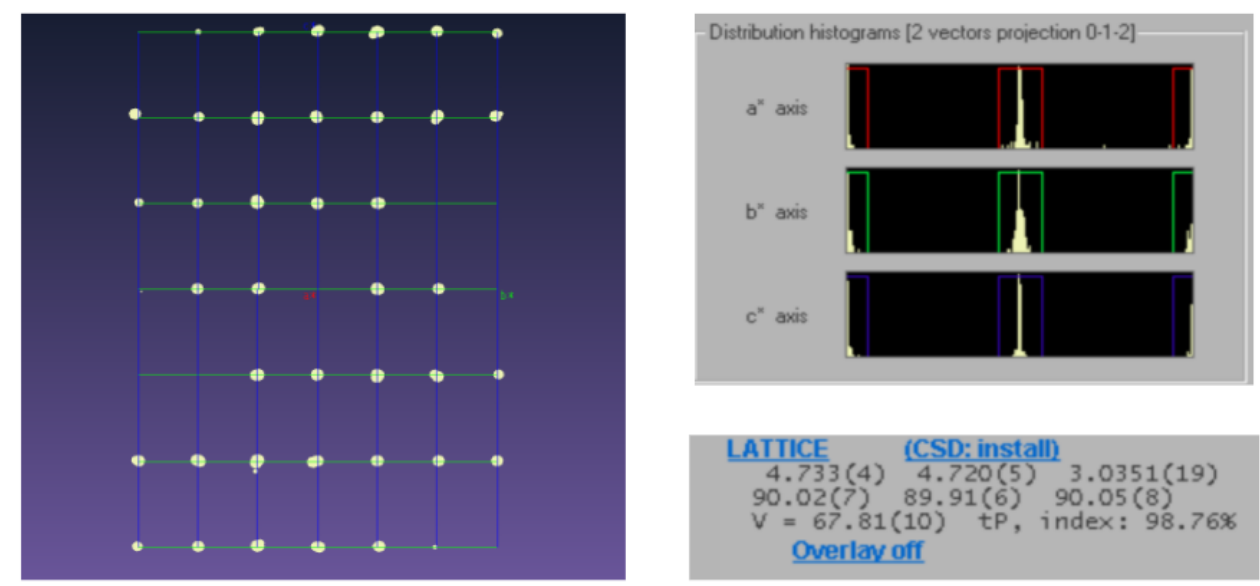

Figure. 4 Detection of polymorphism in $\mathrm{TiO}_{2}$ It was confirmed that anatase and rutile microcrystals can be distinguished by microED. Distribution of reciprocal lattice points and unit cell for (a) anatase and (b) rutile In both cases, the unit cell of the two crystals was determined with very high index rates $(100.0 \%$ and $97.86 \%$ respectively).

\subsection{Small molecules structure determination with molecular replacement}

Of the samples measured in this study, diosgenin was the only sample for which initial phases could not be determined by direct methods. About $80 \%$ of the macromolecular structures deposited in PDB have been determined by molecular replacement and several microED structures were determined by molecular replacement (McCoy et al., 2007). As a test of feasibility, we also investigated whether initial phases for diosgenin could be determined by the molecular replacement method. As a result, initial phases for diosgenin were determined successfully. As mentioned above, it is very difficult to determine the initial phases for datasets with a resolution lower than $1.2 \AA$ by direct methods. On the other hand, datasets with moderate resolution, between 2.0 and $1.2 \AA$, 
are often observed. For small crystals of this quality, the molecular replacement method can be a very effective tool. Furthermore, single atom molecular replacement (McCoy et al., 2017) may also be useful for initial phase determination when no crystal structure is available as a search model. For compounds whose structure has not been determined, even just obtaining the three dimensional structures of non-hydrogen atoms can be important information, so the molecular replacement method may come into use to determine the structure of small molecule compounds in the future.

\section{Conclusion}

In this paper, we demonstrated the usefulness of a newly developed, microED diffractometer by performing crystal structure analysis of several compounds. The ability to routinely measure diffraction data on crystals smaller than $1 \mu \mathrm{m}$ is expected to accelerate structure determination for compounds that have not been determined by X-ray crystallography. It is difficult to determine initial phases using direct methods for compounds of high-molecular weight. In this case, it is worth trying molecular replacement. MicroED is expected to be a quite effective tool not only for crystallographers but also for researchers in material science, organic synthesis, and drug discovery, who need molecular structures. Furthermore, as indicated by the two types of the unit cells of $\mathrm{TiO}_{2}$ in this study, the crystal polymorphs can be easily detected by microED. This is an important capability in the field of drug discovery, especially in the study of physical properties required in drug formulations. This also shows that microED will enable not only simple structure determination, but also analysis that is not possible with conventional powder X-ray diffraction. Quantitative analysis, such as identification of the trace amount of impurity, will also be widely performed with microED (Jones et al., 2018) . In order to perform these experiments using microED routinely, it is essential to use a diffractometer such as the one we have developed in this study to perform the experiments routinely.

While microED has been shown to be a very powerful tool for structure determination, there are still some challenges to be overcome. The first point is the multiple scattering (also known as a dynamical effect) and reduction of intensity of the primary beam because electrons interact much stronger with materials than X-rays (Uyeda, 1968) . In fact, the crystal structures obtained in this study displayed poorer statistics compared to the general statistics obtained with X-rays. Even in the case of X-ray analysis, the dynamical effect becomes critical for highly ordered crystals. However, the primary and secondary extinction in X-ray diffraction is within the range correctable by assuming the extinction length, in particular using the EXTI instruction in SHELXL. The EXTI instruction was used for all the compounds in this study. However, since the dynamical effect in electron diffraction is quite large, the introduction of extinction length is insufficient to model dynamical diffraction. Palatinus et al. described a complex relationship between crystal orientation and thickness on the reflection intensity to correct for multiple scattering (Lukáš Palatinus et al., 
2015) . Furthermore, they introduced some special parameters and showed that multiple scattering can be successfully corrected. This method was implemented in PETS, DynGo, and Jana2006 (L. Palatinus, 2011; Petrícek et al., 2014) . However, in the near future, we are planned to link CrysAlis ${ }^{\text {Pro }}$ ED and Jana2006. The second issue is the determination of absolute structure, which can be performed by using anomalous diffraction. X-ray structure determination, but not in electron diffraction. Brázda et al. proposed a method to determine absolute structures using multiple scattering (Brázda et al., 2019) . Although microED currently has these problems, this method will be widely used as a new option in addition to X-ray, NMR, MS, and other structure determination methods. It is expected that research and development in this area will become brisk, including the continuous development of microED instruments, measurement methods, and programs for structure refinement considering dynamical effect. In the near future, it will be routine for researchers to use X-ray crystallography for crystals above $1 \mu \mathrm{m}$, and microED for those below $1 \mu \mathrm{m}$, using these two techniques in a complementary manner for structural studies (Figure 5). 


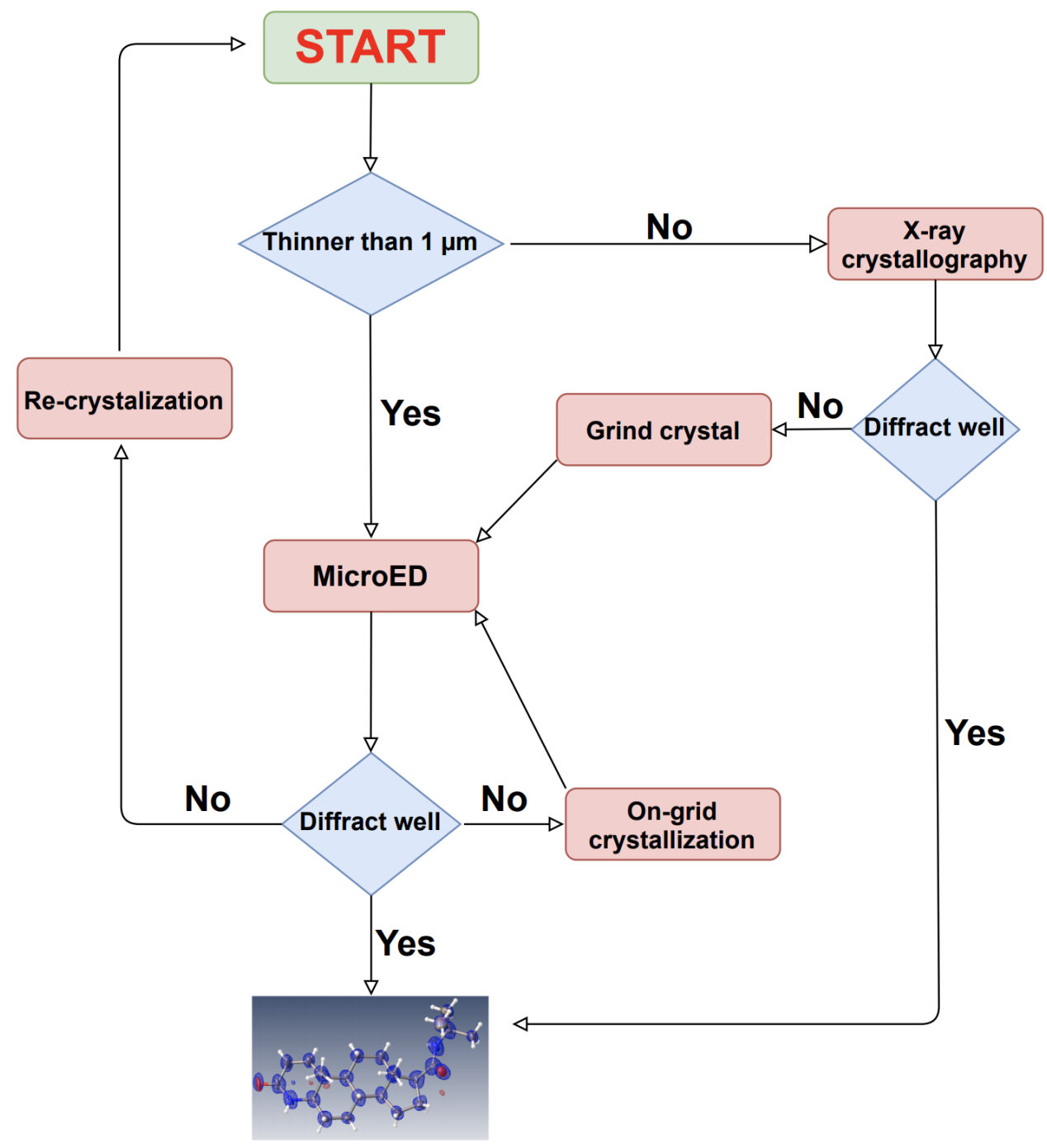

Figure. 5 A comprehensive strategy for structure determination

In case the crystal size is large, structure determination is first attempted using the conventional method of X-ray crystallography. If the crystal size is too small, or if high resolution diffraction points cannot be obtained by X-ray diffraction, then microED is used to determine the structure. These two methods are used in a complementary manner to determine crystal structures.

Associated content

CCDC 2097594 = Cytidine (CIF)

CCDC 2097595 = D-glucose $($ CIF $)$

CCDC $2097596=2,4,6$-Tri(4-pyridyl)-1,3,5-triazine (CIF)

CCDC $2097597=1,3,5$-Triphenylbenzene (CIF)

CCDC $2097598=\mathrm{K}_{2} \mathrm{PtCl}_{4}$ (CIF)

CCDC 2097599 = Acetaminophen (CIF)

CCDC $2097600=$ Gefitinib (CIF)

CCDC $2097601=$ L-tyrosine (CIF)

CCDC 2097602 = Rubrene $($ CIF $)$ 
CCDC $2097603=$ ZIF-8 (CIF)

CCDC 2097604 = Florasulam (CIF)

CCDC 2097605 = iron(II) phthalocyanine bis(pyridine) complex (CIF)

CCDC 2097606 = Finasteride (CIF)

\section{References}

Brázda, P., Palatinus, L., \& Babor, M. (2019). Electron diffraction determines molecular absolute configuration in a pharmaceutical nanocrystal. Science, 364(6441). https://doi.org/10.1126/science.aaw2560

Hoshino, M., Khutia, A., Xing, H., Inokuma, Y., \& Fujita, M. (2016). The crystalline sponge method updated. IUCrJ, 3. https://doi.org/10.1107/S2052252515024379

Inokuma, Y., Yoshioka, S., Ariyoshi, J., Arai, T., Hitora, Y., Takada, K., Matsunaga, S., Rissanen, K., \& Fujita, M. (2013). X-ray analysis on the nanogram to microgram scale using porous complexes. Nature, 495(7442). https://doi.org/10.1038/nature11990

Jones, C. G., Martynowycz, M. W., Hattne, J., Fulton, T. J., Stoltz, B. M., Rodriguez, J. A., Nelson, H. M., \& Gonen, T. (2018). The CryoEM Method MicroED as a Powerful Tool for Small Molecule Structure Determination. ACS Central Science, 4(11). https://doi.org/10.1021/acscentsci.8b00760

Kim, L. J., Xue, M., Li, X., Xu, Z., Paulson, E., Mercado, B., Nelson, H. M., \& Herzon, S. B. (2021). Structure Revision of the Lomaiviticins. Journal of the American Chemical Society, 143(17). https://doi.org/10.1021/jacs.1c01729

McCoy, A. J., Grosse-Kunstleve, R. W., Adams, P. D., Winn, M. D., Storoni, L. C., \& Read, R. J. (2007). Phaser crystallographic software. Journal of Applied Crystallography, 40(4). https://doi.org/10.1107/S0021889807021206

McCoy, A. J., Oeffner, R. D., Wrobel, A. G., Ojala, J. R. M., Tryggvason, K., Lohkamp, B., \& Read, R. J. (2017). Ab initio solution of macromolecular crystal structures without direct methods. Proceedings of the National Academy of Sciences of the United States of America, 114(14). https://doi.org/10.1073/pnas.1701640114

Nannenga, B. L. (2020). MicroED methodology and development. Structural Dynamics, 7(1). https://doi.org/10.1063/1.5128226

Nannenga, B. L., \& Gonen, T. (2019). The cryo-EM method microcrystal electron diffraction (MicroED). In Nature Methods (Vol. 16, Issue 5). https://doi.org/10.1038/s41592-019-0395-x 
Palatinus, L. (2011). PETS-program for analysis of electron diffraction data. Institute of Physics of the AS CR:Prague, Czechia.

Palatinus, Lukáš, Petř́iček, V., \& Correâ, C. A. (2015). Structure refinement using precession electron diffraction tomography and dynamical diffraction: Theory and implementation. Acta Crystallographica Section A: Foundations and Advances, 71. https://doi.org/10.1107/S2053273315001266

Peng, L. M. (1999). Electron atomic scattering factors and scattering potentials of crystals. In Micron (Vol. 30, Issue 6). https://doi.org/10.1016/S0968-4328(99)00033-5

Petrícek, V., Dušek, M., \& Palatinus, L. (2014). Crystallographic computing system JANA2006: General features. In Zeitschrift fur Kristallographie (Vol. 229, Issue 5). https://doi.org/10.1515/zkri-2014-1737

Sawaya, M. R., Rodriguez, J., Cascio, D., Collazo, M. J., Shi, D., Reyes, F. E., Hattne, J., Gonen, T., \& Eisenberg, D. S. (2016). Ab initio structure determination from prion nanocrystals at atomic resolution by MicroED. Proceedings of the National Academy of Sciences of the United States of America, 113(40). https://doi.org/10.1073/pnas.1606287113

Schneider, T. R., \& Sheldrick, G. M. (2002). Substructure solution with SHELXD. Acta Crystallographica Section D: Biological Crystallography, 58(10 I). https://doi.org/10.1107/S0907444902011678

Sheldrick, G. M. (1990). Phase annealing in SHELX-90: direct methods for larger structures. Acta Crystallographica Section A, 46(6). https://doi.org/10.1107/S0108767390000277

Sheldrick, George M. (2015a). Crystal structure refinement with SHELXL. Acta Crystallographica Section C: Structural Chemistry, 71. https://doi.org/10.1107/S2053229614024218

Sheldrick, George M. (2015b). SHELXT - Integrated space-group and crystal-structure determination. Acta Crystallographica Section A: Foundations of Crystallography, 71(1). https://doi.org/10.1107/S2053273314026370

Shi, D., Nannenga, B. L., De La Cruz, M. J., Liu, J., Sawtelle, S., Calero, G., Reyes, F. E., Hattne, J., \& Gonen, T. (2016). The collection of MicroED data for macromolecular crystallography. Nature Protocols, 11(5). https://doi.org/10.1038/nprot.2016.046

Uyeda, R. (1968). Dynamical effects in high-voltage electron diffraction. Acta 
Crystallographica

https://doi.org/10.1107/S0567739468000240
A,

24(1). 